\title{
Compliance of Computed Tomography Scan Requests in Sub-Saharan Africa
}

\author{
Zakari Nikièma ${ }^{1,2}$, Kofi-Mensa Savi de Tové3, Issiaka Sombié2, \\ San Désiré Constantin Barro' ${ }^{1}$, Ziemlé Clément Méda ${ }^{2}$, Rabiou Cissée \\ ${ }^{1}$ Medical Imaging Unit, Souro Sanou University Teaching Hospital, Bobo-Dioulasso, Burkina Faso \\ ${ }^{2}$ Advanced Institute of Health Sciences, Polytechnic University of Bobo-Dioulasso, Bobo-Dioulasso, Burkina Faso \\ ${ }^{3}$ Department of Radiology, Faculty of Medicine, University of Parakou, Parakou, Republic of Benin \\ ${ }^{4}$ Medical Imaging Unit, Yalgado Ouedraogo University Teaching Hospital, Ouagadougou, Burkina Faso \\ Email: nikiemazakari@yahoo.fr
}

Received 1 January 2016; accepted 12 June 2016; published 15 June 2016

Copyright (C) 2016 by authors and Scientific Research Publishing Inc.

This work is licensed under the Creative Commons Attribution International License (CC BY).

http://creativecommons.org/licenses/by/4.0/

cc) (i) Open Access

\begin{abstract}
Objective: To investigate the compliance of computed tomography (CT) scan requests in the X-ray units of Sub-Saharan Africa. Methods: Multi-centric, exhaustive, descriptive and cross-sectional study (Burkina Faso and Benin) focused on CT scan requests implemented and interpreted from May 1 to July 31, 2014. Request compliance was assessed according to the criteria of the French High Authority for Health to which was added a criterion for fulfillment of description of patient clinical information. An exam was deemed compliant when all criteria were met. Ratios were compared by means of $\mathrm{Chi}^{2}$ test at $0.05 \%$ significance level. Results: Among the 426 colligated computed tomography prescriptions, the least filled fields were allergy concept $(0 \%)$, creatininemia $(0.3 \%)$, examination purpose $(50.2 \%)$. The overall compliance rate was $35 \%$. The requests made by medical specialists were more compliant than those made by general practitioners and students $(p=0.003)$. Fulfillment of clinical information description was also better among medical specialists compared to the other prescribers $(p=0.002)$. Conclusion: It is important to improve the quality of computed tomography (CT) scan requests in the hospitals of Bobo-Dioulasso (Burkina Faso) and Parakou (Benin).
\end{abstract}

\section{Keywords}

Compliance, Prescription, Scanner, Sub-Saharan Africa

\section{Introduction}

Radiology examination request is a common operation performed every day by qualified health workers. It is a

How to cite this paper: Nikièma, Z., de Tové, K.-M.S., Sombié, I., Barro, S.D.C., Méda, Z.C. and Cissé, R. (2016) Compliance of Computed Tomography Scan Requests in Sub-Saharan Africa. Open Journal of Radiology, 6, 125-130.

http://dx.doi.org/10.4236/ojrad.2016.62019 
diagnostic test clinicians require to support or confirm a diagnostic hypothesis, to guide an intervention and monitor the efficiency of a therapeutic method. They expect from the examinations performed and information collected the possibility of reducing the margin of uncertainty which is commonly associated with clinical practice [1]. That is why that request should be governed by specific rules [1]-[3]. It actually exposes the medical radiologist to responsibility [3]. Shortcomings are identified in the writing of X-ray or radiological examination requests with a very variable compliance rate in the literature ranging from $31 \%$ to $56.4 \%$ [4] [6] [8]. Besides, in the international literature, the subject is poorly documented [1]-[11] and to the best of our knowledge only two studies of this kind have been conducted in Africa [6] [7]. Scanner had been introduced in Burkina Faso and Benin for about 10 years and no analysis of the request for this medical exam had been done. The purpose of the current study was to improve the prescription of this medical exam in our hospitals. Its aim was to investigate on the compliance of CT scan requests collected in the radio-diagnostic and medical imaging units.

\section{Materials and Methods}

We carried out a cross-sectional study with descriptive purpose on all the CT scan requests submitted to the medical imaging unit of the Souro Sanon Hospital of Bobo-Dioulasso (Burkina Faso) and to the "Santa Maria" Medical Imaging Centre of Parakou (Benin) from May 1 to July 31, 2014. Regarding the determination of our sample size, as we do not have a previous rate of CT scan requests compliance, we estimated it at $50 \%$ to get a maximum size through the formula $\mathrm{n}=\epsilon \times \mathrm{p}(1-\mathrm{p}) / \mathrm{e}^{2}$. The minimum size was 384 prescriptions but due to problems of data quality, we decided to increase our sample by $10 \%$ so as to address possible dismissals of request forms; as a result we got a sample of 426 requests. All the CT scan prescriptions performed and logged in the protocol were included till reaching the estimated sample size.

Data were collected from CT scan request documents while respecting anonymity. The scan compliance variables defined by the French High Authority for Health (HAS) to which we added patient sex, were gathered [4]. One mark was allocated depending on the presence of the following information: prescription date; prescriber full name; requesting/referring unit; patient full name; patient sex; patient age or date of birth; anatomical region to be explored; patient clinical information; CT scan purpose or aim.

Request forms were classified into four groups according to the following score: group 1 ( 0 to 6 marks); group 2 (7 marks); group 3 (8 marks); group 4 (9 marks).

Moreover, we added criteria for fulfillment of patient clinical information description based on: mode of signs onset and/or context of occurrence; short description of clinical presentation; reference to a concept of occupational disease; syndromic surveillance summary; patient past health history; recent or previous contributing paraclinical results; and date of onset and/orduration or evolutionary profile.

Data were entered and processed on micro-computer using Epi info software version 3.5.1. Graphs were created in Microsoft Excel 2007. Ratios were compared through $\mathrm{Chi}^{2}$ test with a 5\% significance threshold.

\section{Results}

Four hundred twenty six (426) request forms including 244 (57.28\%) from the medical imaging unit of the Souro Sanon Hospital of Bobo-Dioulasso (Burkina Faso) and 182 (42.72\%) from the "Santa Maria" Medical Imaging Center of Parakou were analyzed during the study period.

In $31.20 \%$ of the cases they were prescribed by a medical specialist, in $29.80 \%$ of the cases by a junior medical intern and in $22.10 \%$ of the cases by a general practitioner. In $16.9 \%$ of the cases, prescriber's qualification was not specified.

Figure 1 indicates the level of information completion of the different fields of a request. The fields which are more commonly not reported were allergy concept (100\%), creatininemia (99.7\%), purpose (49.8\%), requester first name (47.7\%) and patient age (31.7\%).

The levels of request information filling according to requester qualification are defined in Table 1. Among the criteria for request compliance assessment, the least filled one among medical specialists was patient age which was not reported in $37.6 \%$ of the cases. The request purpose was the least completed field among general practitioners; not recorded in $47.9 \%$ of the cases. As regards junior medical interns, requester first name was not specified in $99.2 \%$ of the cases and purpose in $66.2 \%$ of the cases. Among prescribers whose qualification was unknown, the least filled fields were requester name, request purpose and requester first name which were not mentioned respectively in $54.2 \%, 73.6 \%$ and $91.7 \%$ of the cases. There was a statistically significant difference 


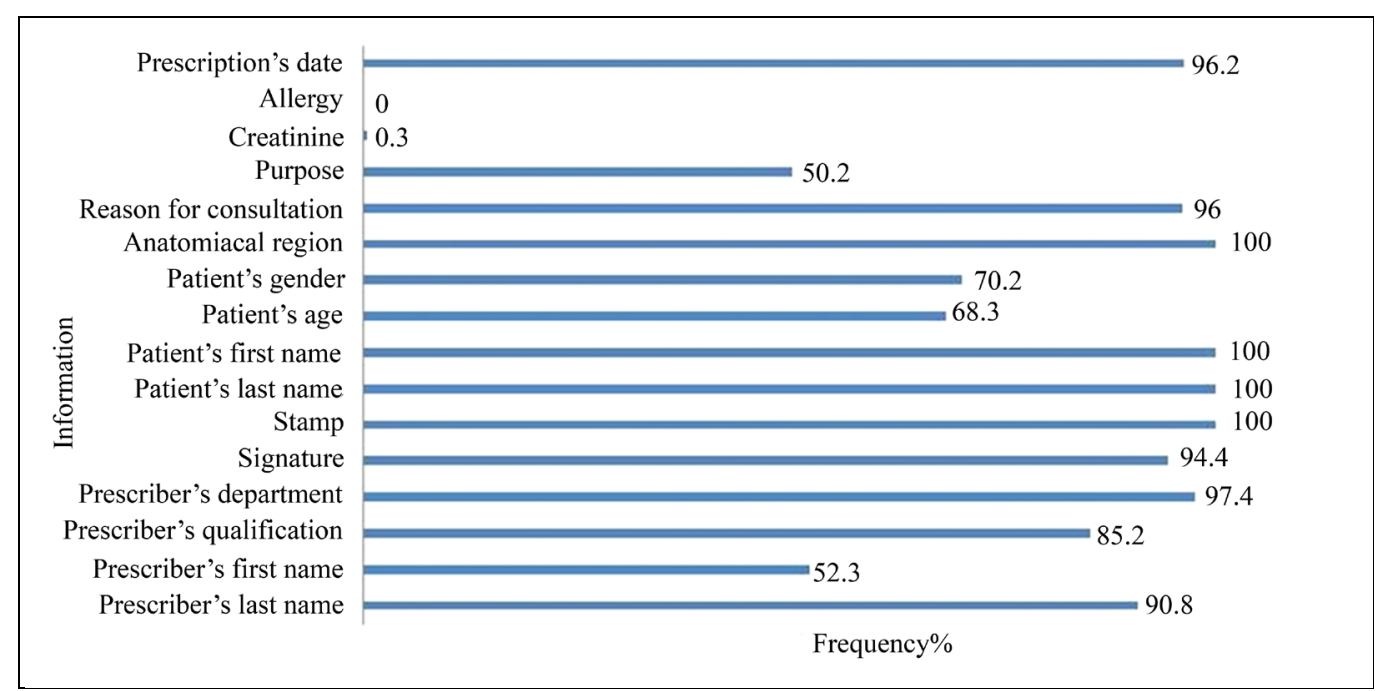

Figure 1. Summary of overall level of request field filling.

Table 1. Distribution of request filling levels according to requester qualification.

\begin{tabular}{|c|c|c|c|c|}
\hline Qualification & $\begin{array}{l}\text { Medical specialist } \\
\text { (\%) }\end{array}$ & $\begin{array}{c}\text { General practitioner } \\
\text { (\%) }\end{array}$ & Junior intern (\%) & Unknown (\%) \\
\hline Indicationof requestername & 100 & 100 & 100 & 45.8 \\
\hline Indication of requester first name & 100 & 88.3 & 0.8 & 8.3 \\
\hline Indicationof unit name & 95,5 & 96.8 & 100 & 97.2 \\
\hline Presenceof signature & 100 & 92.5 & 96.8 & 82 \\
\hline Presenceof stamp & 100 & 100 & 100 & 100 \\
\hline Mentionof patientname & 100 & 100 & 100 & 100 \\
\hline Mention of patient first name & 100 & 100 & 100 & 100 \\
\hline Indicationof patientage & 62,4 & 75.5 & 77.2 & 54.2 \\
\hline Indication of patientsex & 90,2 & 66.3 & 63.5 & 60.8 \\
\hline Indication ofanatomicalregion & 100 & 100 & 100 & 100 \\
\hline Indication of reason & 97 & 97.9 & 97.6 & 88.9 \\
\hline Indication of a valid reason & 85,7 & 69.1 & 56 & 30.5 \\
\hline Indication of purpose & 77,4 & 52.1 & 33.8 & 26.4 \\
\hline Indication of creatininemia & 4,5 & 3.2 & 2.4 & 0 \\
\hline Indication of an allergy concept & 0 & 0 & 0 & 0 \\
\hline Indication of date & 100 & 100 & 98.4 & 80.5 \\
\hline
\end{tabular}

between filling rates of purpose field by medical specialists and by other categories of prescriber $(p=0.004)$.

Fulfillment of patient clinical information description according to requester qualification is illustrated in Figure 2. The difference of ratio was statistically significant between medical specialists and others categories of prescribers $(p=0.002)$. The compliance score had indicated a ratio of compliant requests (group 4) estimated at 35\% of the cases followed by group 3 (43.9\%), group 2 (16.2\%) and group 1 (4.9\%). Figure 3 shows the distribution of prescriber qualification according to compliance scores. As a result, compliant requests (group 4) were more common among medical specialists $(57.9 \%)$, with a significant difference $(p=0.003)$ compared to those of the other prescribers. 


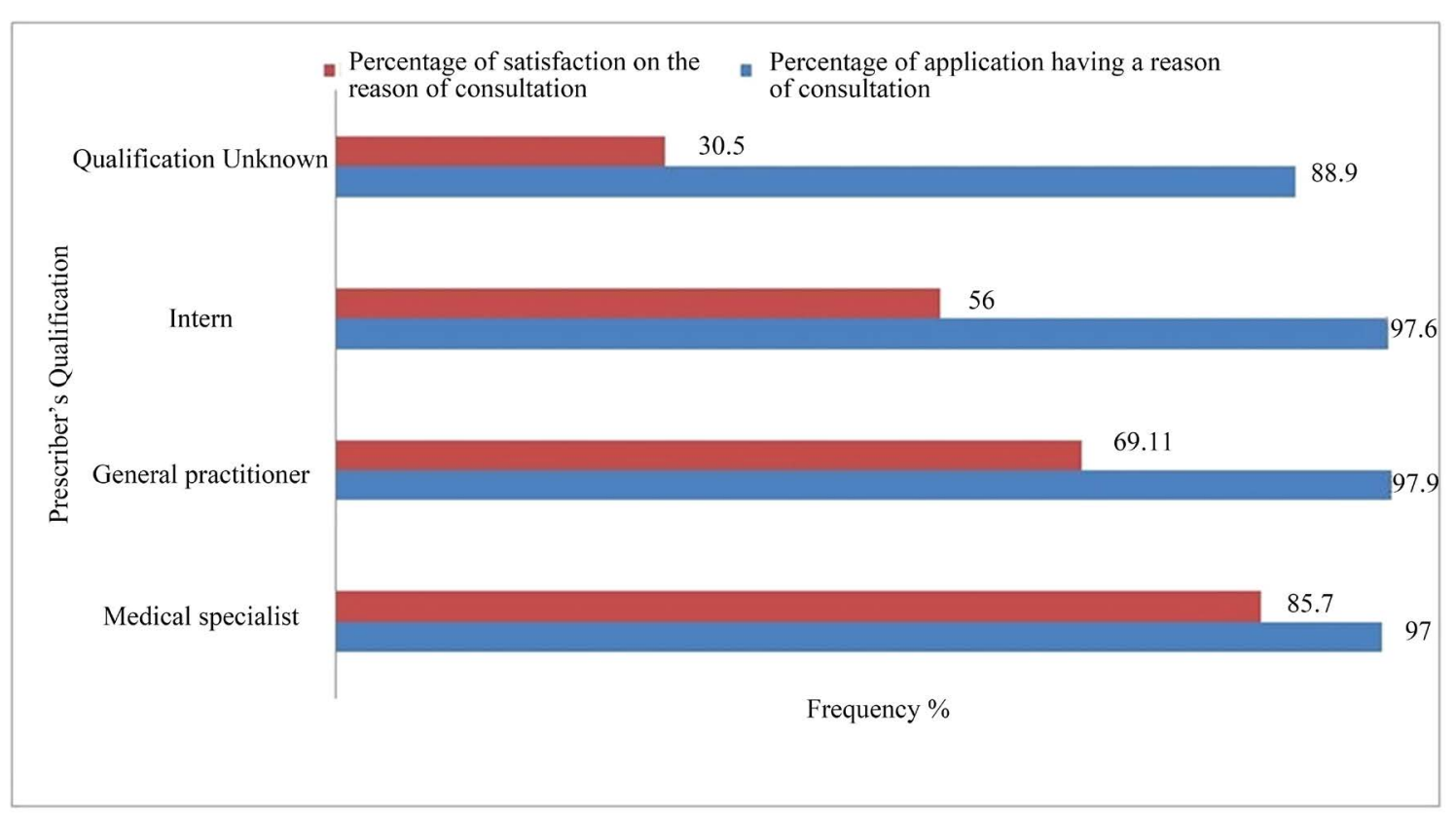

Figure 2. Fulfillment of description of patient clinical condition according to requester qualification.

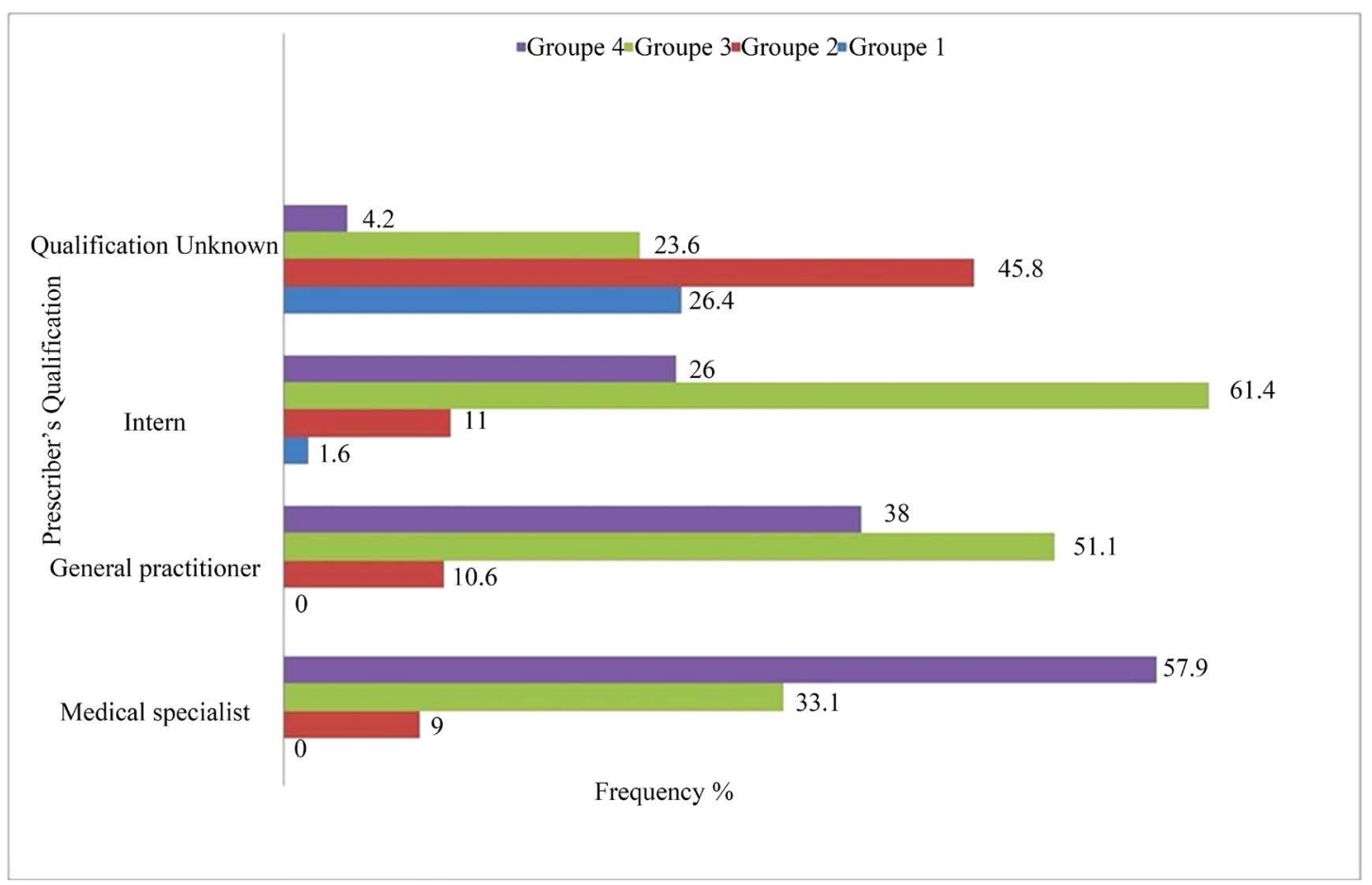

Figure 3. Distribution of compliance score according to prescriber qualification.

\section{Discussion}

This study suggests a low compliance rate of CT scan requests in our hospitals. This low rate is mainly the result of lack of indication of purpose, patient age, prescriber first name, concept of allergy to products and creatinemia. It was also noted that compliance rate and fulfillment of description of patient clinical condition were better in medical specialists compared to the other prescribers.

CT scan compliance rate noted in this research work was similar to the one reported in the study conducted by 
the French High Authority for Health [4].

CT scan compliance and satisfaction of description of patient clinical condition were better among medical specialists compared to the other categories of prescriber. This may be due to the gradient of skills and knowledge between those different prescribers. The most qualified specialists have a better knowledge of CT scan contribution. As a whole, these results must lead to provide capacity building for all the stakeholders in terms of $\mathrm{x}$-ray exam prescription since examination compliance remains low even among specialists (57.5\%). In France, standardization and computerization of examination coupons/orders helped increase the compliance rate to $100 \%$ [8].

Poor completion of information on purpose, patient age, prescriber first name, concept of allergy to products and creatinemia were also reported in other studies [6]-[8].

In the HAS research work [4] patient age was the least filled field (5.9\%); this is lower than the $25.09 \%$ in Cameroun [7] and 31.7\% reported in our study. This gap may be due to the fact that in Burkina Faso and Benin, many people ignore their date of birth and do not have any document likely to provide that information, such as civil status documents during medical consultations.

Gbazi et al. [6] had noted a lack of prescriber identification in 2.5\% of the cases in Côte d'Ivoire. In France, HAS [4] pointed out a lack of information on name in scanner prescription in $11.8 \%$ of the cases. In this research work, if prescriber name was not mentioned in $10 \%$ of the cases, absence of prescriber first name was the most important. This may lead to confusion since one knows that often in the same units there are prescribers bearing the same surnames.

In our study, request reason and purpose were filled respectively in $96 \%$ and $50.2 \%$ of the cases. These results are close to those of HAS [4], with 94.5\%, and 71.1\% respectively. By contrast, in Côte d'Ivoire Gbazi et al found out a completion rate of reason lower than ours; 44\% [6]. As well, the exam purpose mentioned in the form of clinical hypothesis was specified in this research work only in $32.2 \%$ of the cases; this was similar to the 34\% encountered by Gbazi et al. [6].

The good characterization of computed tomography of an abnormality may require injection of radio contrast medium; this requires making sure that patient kidney works well by measuring creatinemia. In this study, that measure was mentioned only on $2.8 \%$ of the requests. In contrast, in the study carried out by Girard et al. in 2007, it was reported only in $87.5 \%$ of the cases [8]. The administration of an iodinated contrast mediumis not systematic during a CT scan. That is why this information and the one on allergy concept were not taken into account in the calculation of request compliance scores. However, the systematic addition of kidney function test in scanner prescription may be necessary in order to prevent a potential partial postponement of the exam for a subsequent complement of CT scan series with injection. Actually, the possibility of kidney failure represents a contraindication. Notification of creatinemia is compulsory for subjects above 65 years of age, diabetic patients and subjects with kidney failure [8]. The allergy concept had not been reported in any of our patients. This information was available in only 3.36\% of the cases in the study of Girard et al. [8].

Based on the results obtained, it can be claimed that this study contributes to a better knowledge of compliance of CT scan requests in Burkina Faso and Benin. They will be helpful for developing actions to improve prescription of X-ray exams by physicians in Sub-Saharan Africa's hospitals.

\section{Conclusion}

This research work has revealed a common non-compliance of CT scan request forms. As a result, this leads to possible postponement of exam, difficulty in exam performance and interprétation. The current study suggests capacity building for all the stakeholders in the area of X-ray exam prescription. Assiduous attendance during initial training program for interns, standardization and computerization of exam requests could represent solutions.

\section{Conflict of Interest}

None

\section{Authors' Contribution}

All the authors contributed to this research work. They read and agreed to the final version of the manuscript. 


\section{References}

[1] Roussel, P. and Lelievre, N. (2002) Improving the Quality of Medical Imaging Prescriptions. Diagnostic and Interventional Imaging, 83, 621-625.

[2] French High Authority for Health (Haute Autorité de la Santé) (2005) National Agency for Certification and Assessment in Health Issues. Guide to Medical Imaging Good Practices. HAS-Anaes, Paris.

[3] Thibierge, M., Fournier, L. and Canabis, E.A. (1999) Medical Responsibility Principles and Medical Imaging Practice. Diagnostic and Interventional Imaging, 80, 701-707.

[4] French High Authority for Health (Haute Autorité de la Santé) (2010) Indicator of Compliance of Imaging Exam Requests. HAS, Paris.

[5] Boland, G.W.L. (2009) Opinion from Herding Cats Herding Cats Toward Best Practices: Standardizing the Radiology Work Process. American Journal of Roentgenology, 193, 1593-1595. http://dx.doi.org/10.2214/AJR.09.2885

[6] Gbazi, G.C., Abby, B.C., Konan, A.V., N’Dri, K., Aké, A.C., Konan, A.C. and Kouassi, B. (2006) Analysis of X-Ray Exam Request Forms at the Cocody University Teaching Hospital: Initial Results of 1446 Requests. Diagnostic and Interventional Imaging, 87, 1425-1426.

[7] Moifo, B., Kamgnie, M.N., Fointama, N.F., Tambe, J., Tebere, H. and Fotsin, J.G. (2014) Évaluation de la conformité des demandes d'examens d'imagerie médicale: Une expérience en Afrique subsaharienne. Médecine et Santé Tropicales, 24, 392-396.

[8] Girard, D., Dozol., A, Martinez, F., Montagne, B., Houdart, E., Laredo, J.D., Soyer, P. and Segouin, C. (2011) Compliance and Quality of X-Ray Exam Requests. Journal of Epidemiology and Public Health, 59, S51.

[9] Broder, J. and Warshauer, D.M. (2005) Increasing Utilization of Computer Tomography in the Adult Emergency Department, 2000-2005. Emergency Radiology, 1, 25-30.

[10] Bruel, J.M., Valette, P.J. and Rhamouni, A. (2009) Medical Imaging Request: Key Factor for Improving Patient Care Quality. Diagnostic and Interventional Imaging, 90, 1486-1487.

[11] Brink, J.A. (2001) Opinion. Professional Practice Performance: Is “Good” Enough? American Journal of Roentgenology, 197, 1288-1290. http://dx.doi.org/10.2214/AJR.11.7605 